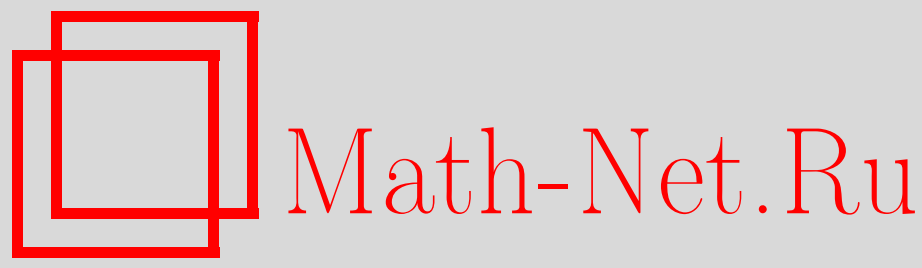

П. В. Ролдугин, А. В. Тарасов, Функции без коротких имплицент. Часть II: методы построения, Дискрет. матем., 2015, том 27, выпуск 4, 120-132

DOI: https://doi.org/10.4213/dm1351

Использование Общероссийского математического портала Math-Net.Ru подразумевает, что вы прочитали и согласны с пользовательским соглашением http://www.mathnet.ru/rus/agreement

Параметры загрузки:

IP : 54.210 .77 .194

26 апреля 2023 г., 16:34:17

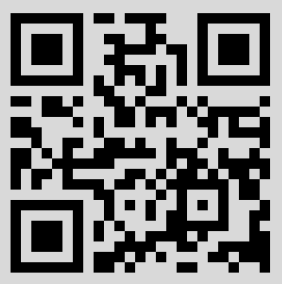




\title{
Функции без коротких имплицент. Часть II: методы построения
}

\author{
() 2015 г. П. В. Ролдугин*, А. В. Тарасов**
}

Статья является продолжением работы "Функции без коротких имплицент. Часть I: нижние оценки весов". Во второй части предложены различные методы построения булевых функций от $n$ переменных, не имеющих имплицент от $k$ переменных. Первый из предложенных методов базируется на градиентном алгоритме, второй и третий методы используют определенное комбинаторное правило построения, четвертый метод основан на случайном выборе элементов носителя функции. В зависимости от значения $k$ методы имеют различную эффективность. Выводятся верхние оценки минимального значения $w(n, k)$ весов построенных функций. Вместе с нижними оценками величины $w(n, k)$ из первой части статьи это позволяет получить асимптотически точную оценку вида $w(n, k)=\Theta(\ln n)$ при $n \rightarrow \infty$.

Ключевые слова: булевы функции, имплиценты, методы построения функций, вес булевой функции

Введение. Введение и краткий обзор известных результатов по рассматриваемой проблематике содержится в статье [1]. Приведем основные определения и результаты указанной статьи, на которые опирается настоящая работа.

Имплицентой булевой функции $f\left(x_{1}, \ldots, x_{n}\right)$ называется такая отличная от константы булева функция $g\left(x_{i_{1}}, \ldots, x_{i_{k}}\right)$, что для любого набора $\left(\alpha_{1}, \ldots, \alpha_{n}\right) \in V_{n}$ выполняется равенство $f\left(\alpha_{1}, \ldots, \alpha_{n}\right) \cdot g\left(\alpha_{i_{1}}, \ldots, \alpha_{i_{k}}\right)=f\left(\alpha_{1}, \ldots, \alpha_{n}\right)$ (см. [2]). Если все переменные $x_{i_{1}}, \ldots, x_{i_{k}}$ являются существенными переменными функции $g$, то $k$ называется длиной имплиценты. Функция $w(n, k)$ определяется как минимальное возможное значение веса булевой функции от $n$ переменных, не имеющей имплицент от $k$ или менее переменных. Случай $k=1$ тривиален и разобран в статье [1].

Комбинаторно полной матрицей порядка $k>1$ называется $(0,1)$-матрица размера $m \times n(m$ строк, $n$ столбцов, $n>k)$, если в любой ее подматрице размера $m \times k$ для любого булевого вектора $\vec{v}$ длины $k$ найдется строка, равная $\vec{v}$. В [1] доказано, что, если функция $f\left(x_{1}, \ldots, x_{n}\right)$ принимает значение 1 на наборах

*Место работы: Московский государственный университет информационных технологий, радиотехники и электроники, e-mail: PavRoldugin@rambler.ru

** Место работы: Московский государственный университет информационных технологий, радиотехники и электроники, e-mail: alextar1@yandex.ru 
$\left\{\left(\alpha_{1}^{(i)}, \ldots, \alpha_{n}^{(i)}\right), i=\overline{1,\|f\|}\right\}$, то матрица

$$
\left(\begin{array}{ccc}
\alpha_{1}^{(1)}, & \ldots, \alpha_{n}^{(1)} \\
\alpha_{1}^{(\|f\|)}, & \ldots, \alpha_{n}^{(\|f\|)}
\end{array}\right)
$$

является комбинаторно полной порядка $k>1$ тогда и только тогда, когда у функции $f$ нет имплицент длины $k$. Отсюда выведено, что при фиксированном значении $n$ и $k, n>k>1$, величина $w(n, k)$ равна минимальному из возможных значений числа строк комбинаторно полной матрицы порядка $k$, имеющей ровно $n$ столбцов.

Ранее (см. [1]) были получены нижние оценки функции $w(n, k)$. Как видно из приведенных результатов, верхние оценки этой функции можно получить путем построения комбинаторно полных матриц порядка $k$ с $n$ столбцами. Собственно число строк в такой матрице и будет верхней оценкой $w(n, k)$. Далее статья посвящена методам построения указанных матриц.

Кратко охарактеризуем предлагаемые ниже алгоритмы построения комбинаторно полных матриц. В первом параграфе проблема построения комбинаторно полной матрицы сводится к известной задаче нахождения покрытия столбцов булевой матрицы строками ([3], [4]); результат решения такой задачи "жадным" алгоритмом дает наилучшую из полученных на данный момент верхних оценок: $w(n, k) \leqslant 2^{k} \cdot k \cdot \ln n$. Вместе с нижними оценками величины $w(n, k)$ из [1] это позволяет доказать асимптотически точную оценку: $w(n, k)=\Theta(\ln n)$ при $n \rightarrow \infty$, то есть существование таких $C_{1}, C_{2}>0$ и такого $n_{0}$, что для всех $n>n_{0}$ верно соотношение $C_{1} \cdot \ln n<w(n, k)<C_{2} \cdot \ln n$.

Однако при сведении поставленной в статье задачи к задаче о покрытии ее размер настолько возрастает, что практическое применение предложенного алгоритма затруднительно даже при небольших значениях $n$ и $k$. Кроме того, при значениях $k$, близких к $n$, получаемая оценка хуже тривиальной оценки $w(n, k) \leqslant 2^{n}$ (матрица с $n$ столбцами, содержащая все возможные $2^{n}$ различных строк, очевидно является комбинаторно полной порядка $k$ для любого значения $1<k<n)$. Учитывая указанные недостатки "жадного" алгоритма, во втором параграфе указан способ построения комбинаторно полной матрицы, дающий оценку $w(n, k) \leqslant \sum_{t=1}^{t_{0}}\left(\begin{array}{c}n \\ n-k+1\end{array}\right)$, где $t_{0}=\left\lceil\frac{1+k}{n-k+1}\right\rceil$. Алгоритм строит матрицу построчно, поэтому трудоемкость построения матрицы пропорциональна указанной оценке. По сравнению с "жадным" алгоритмом, верхняя оценка, приведенная во втором параграфе, лучше в случаях, когда $k$ близко к $n$.

В третьем параграфе предложен метод построения комбинаторно полных матриц порядков 2 и 3. Получаемая из этого метода верхняя оценка величин $w(n, 2)$ и $w(n, 3)$ заметно лучше, чем оценка в общем случае, и вплотную приближается к нижним оценкам, доказанным в [1].

Наконец, в последнем параграфе рассмотрен вероятностный метод построения комбинаторно полной матрицы - алгоритм, в котором строки матрицы выбираются случайно, независимо и равновероятно. В предположении, что число строк равно оценке для "жадного" алгоритма, т.е. $2^{k} \cdot k \cdot \ln n$, при различных предположениях 
о поведении $k$ ( $k$ - фиксированное число; $k=c \cdot n, c<1 ; k=n-d, d$ - фиксированное) доказано, что вероятность получить комбинаторно полную матрицу порядка $k$ стремится к 1 при $n \rightarrow \infty$. Неформально говоря, из этого следует, что можно вместо "жадного" алгоритма применять гораздо менее трудоемкий вероятностный метод, а число строк в полученной матрице будет фактически таким же.

1. "Жадный" алгоритм построения. В данном параграфе рассмотрим подход к построению комбинаторно полных матриц, который позволит получить верхнюю оценку величины $w(n, k)$. Однако указанный подход имеет очень высокие требования по памяти и трудоемкости, что не позволяет использовать его на практике для получения комбинаторно полных матриц, содержащих хотя бы несколько десятков столбцов.

Рассмотрим конструкцию, которая позволит переформулировать понятие комбинаторной полноты матриц в терминах покрытия булевых матриц. Вслед за [3] назовем в $(0,1)$-матрице $D=\left(d_{i, j}\right)$ строку $\vec{D}_{i}$ и столбец $D_{j}^{\downarrow}$ пересекающимися, если $d_{i, j}=1$. Покрытием матрицы $D$ называется такой набор ее строк $\vec{D}_{s_{1}}, \ldots, \vec{D}_{s_{t}}$, что для любого столбца $D_{j}^{\downarrow}$ в этом наборе найдется пересекающаяся с ним строка.

При фиксированных значениях $n$ и $k, n>k \geqslant 2$, определим $(0,1)$-матрицу $T(n, k)$ следующим образом. Строки этой матрицы проиндексируем (пометим) всеми различными $n$-мерными булевыми векторами $\left(\alpha_{1}, \ldots, \alpha_{n}\right) \in V_{n}$, всего строк в матрице $T(n, k)-2^{n}$. Столбцы матрицы $T(n, k)$ проиндексируем упорядоченными парами $\left(\left(\beta_{1}, \ldots, \beta_{k}\right) ;\left(i_{1}, \ldots, i_{k}\right)\right)$, где $\left(\beta_{1}, \ldots, \beta_{k}\right) \in V_{k}$ и $1 \leqslant i_{1}<i_{2}<$ $\ldots<i_{k} \leqslant n$; столбцов в матрице $T(n, k)$ будет ровно $2^{k}\left(\begin{array}{l}n \\ k\end{array}\right)$. На пересечении строки, соответствующей вектору $\left(\alpha_{1}, \ldots, \alpha_{n}\right)$, и столбца, соответствующего паре $\left(\left(\beta_{1}, \ldots, \beta_{k}\right) ;\left(i_{1}, \ldots, i_{k}\right)\right)$, стоит единица тогда и только тогда, когда $\alpha_{i_{1}}=\beta_{1}, \alpha_{i_{2}}=$ $\beta_{2}, \ldots, \alpha_{i_{k}}=\beta_{k}$. Заметим, что число единиц в каждой строке матрицы $T(n, k)$ равно $\left(\begin{array}{l}n \\ k\end{array}\right)$, поскольку со строкой, помеченной $\left(\alpha_{1}, \ldots, \alpha_{n}\right)$, пересекаются только столбцы, проиндексированные парами вида $\left(\left(\alpha_{i_{1}}, \ldots, \alpha_{i_{k}}\right) ;\left(i_{1}, \ldots, i_{k}\right)\right)$. С другой стороны, со столбцом, помеченным парой $\left(\left(\beta_{1}, \ldots, \beta_{k}\right) ;\left(i_{1}, \ldots, i_{k}\right)\right)$, пересекаются те и только те строки $\left(\alpha_{1}, \ldots, \alpha_{n}\right)$, для которых $\alpha_{i_{1}}=\beta_{1}, \alpha_{i_{2}}=\beta_{2}, \ldots, \alpha_{i_{k}}=\beta_{k}$, т. е. оставшиеся $n-k$ координат в строке $\left(\alpha_{1}, \ldots, \alpha_{n}\right)$ могут быть любыми. Следовательно, число единиц в каждом столбце матрицы $T(n, k)$ равно $2^{n-k}$.

При помощи матрицы $T(n, k)$ можно получить следующий критерий комбинаторной полноты матрицы.

Утверждение 1. Пусть $m, n, k$ - натуралъные числа, $n>k>1, m \geqslant 2^{k}$. Maтрица А размера $m \times n$, состоящая из строк $\vec{A}_{1}, \ldots, \vec{A}_{m}$, является комбинаторно полной порядка $k$ тогда и толъко тогда, когда строки матрицы $T(n, k)$, помеченные векторами $\vec{A}_{1}, \ldots, \vec{A}_{m}$, образуют покрытие матрицы $T(n, k)$.

Доказательство. По определению матрица $A$ является комбинаторно полной порядка $k$ тогда и только тогда, когда для любого набора чисел $1 \leqslant i_{1}<i_{2}<\ldots<i_{k} \leqslant n$ и любого вектора $\left(\beta_{1}, \ldots, \beta_{k}\right) \in V_{k}$ найдется строка $\vec{A}_{j}=$ $\left(\alpha_{1}^{(j)}, \ldots, \alpha_{n}^{(j)}\right), j \in \overline{1, m}$, матрицы $A$ со свойством: $\alpha_{i_{1}}^{(j)}=\beta_{1}, \ldots, \alpha_{i_{k}}^{(j)}=\beta_{k}$. Но это означает, что в матрице $T(n, k)$ столбец, проиндексированный парой 
$\left(\left(\beta_{1}, \ldots, \beta_{k}\right) ;\left(i_{1}, \ldots, i_{k}\right)\right)$, пересекается со строкой, помеченной вектором $\overrightarrow{A_{j}}$. Поскольку пара $\left(\left(\beta_{1}, \ldots, \beta_{k}\right) ;\left(i_{1}, \ldots, i_{k}\right)\right)$ - любая, постольку каждый столбец в матрице $T(n, k)$ пересекается с некоторой строкой этой же матрицы, помеченной одним из векторов $\vec{A}_{1}, \ldots, \vec{A}_{m}$. Последнее и означает, что строки матрицы $T(n, k)$, проиндексированные векторами $\overrightarrow{A_{1}}, \ldots, \overrightarrow{A_{m}}$, образуют покрытие матрицы $T(n, k)$.

Обратное утверждение доказывается такими же рассуждениями в обратном порядке.

Таким образом, согласно доказанному критерию, задача построения комбинаторно полной матрицы порядка $k$ сводится к процедуре построения покрытия матрицы $T(n, k)$. Для нахождения такого покрытия можно использовать «жадный» принцип, реализуемый градиентным алгоритмом ([4]). Будем строить покрытие, добавляя в него строки одну за другой, руководствуясь следующим правилом: если на некотором шаге уже выбранные строки покрывают некоторое количество столбцов матрицы $T(n, k)$, то добавляем ту строку, которая пересекается с наибольшим числом еще не покрытых столбцов (если таких строк несколько, то выбираем любую). Такой алгоритм построения покрытия булевой матрицы хорошо изучен ([4]). Опираясь на известный результат, докажем следующую оценку.

Утверждение 2. Для натуралъных значений $n, k, n>k>1$ верно неравенство

$$
w(n, k) \leqslant\left\lceil 2^{k} \cdot\left(\ln \left(\begin{array}{l}
n \\
k
\end{array}\right)+1\right)\right\rceil
$$

Доказательство. Воспользуемся теоремой, приведенной, например, в [4]. Если для некоторого $\gamma, 0<\gamma \leqslant 1$, в каждом столбце матрицы $M$, имеющей $p$ строк и $s$ столбцов, содержится не меньше $\gamma \cdot p$ единиц и $\gamma \cdot s \geqslant 1$, то градиентный алгоритм, применяемый к матрице $M$, дает покрытие из не более чем

$$
\left\lceil\frac{1}{\gamma} \cdot \ln (\gamma \cdot s)\right\rceil+\frac{1}{\gamma}
$$

строк, где $\lceil x\rceil$ - наименьшее целое, не меньшее $x$.

В качестве матрицы рассмотрим матрицу $T(n, k)$. Имеем: $p=2^{n}, s=2^{k} \cdot\left(\begin{array}{l}n \\ k\end{array}\right)$, в каждом столбце матрицы содержится ровно $2^{n-k}$ единиц. Поэтому $\gamma=2^{-k}$ и

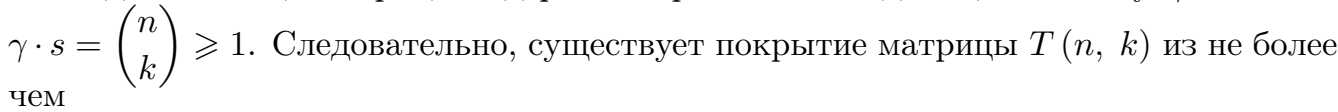

$$
\left\lceil 2^{k} \cdot \ln \left(\begin{array}{l}
n \\
k
\end{array}\right)\right\rceil+2^{k}=\left\lceil 2^{k} \cdot \ln \left(\begin{array}{l}
n \\
k
\end{array}\right)+2^{k}\right\rceil=\left\lceil 2^{k} \cdot\left(\ln \left(\begin{array}{l}
n \\
k
\end{array}\right)+1\right)\right\rceil
$$

строк. Согласно утверждению 1 , это эквивалентно существованию комбинаторно полной матрицы порядка $k$ с не более чем таким количеством строк и $n$ столбцами, то есть $w(n, k) \leqslant\left\lceil 2^{k} \cdot\left(\ln \left(\begin{array}{l}n \\ k\end{array}\right)+1\right)\right\rceil$.

Для случая $k \geqslant 3$ в качестве следствия доказанного утверждения получим более простую оценку. 
Следствие 1. Для натуральных значений $n u k, n>k \geqslant 3$, верно неравенство

$$
w(n, k)<2^{k} \cdot k \cdot \ln n .
$$

Доказательство. Используем очевидные неравенства $\left(\begin{array}{l}n \\ k\end{array}\right)=\frac{n \cdot(n-1) \cdot \ldots \cdot(n-k+1)}{k !} \leqslant \frac{n^{k}}{k !}$ и $\lceil x\rceil \leqslant x+1$. Получаем, что

$$
\begin{gathered}
w(n, k) \leqslant\left\lceil 2^{k} \cdot\left(\ln \left(\begin{array}{l}
n \\
k
\end{array}\right)+1\right)\right] \leqslant 2^{k} \cdot\left(\ln \left(\begin{array}{l}
n \\
k
\end{array}\right)+1\right)+1 \leqslant 2^{k} \cdot\left(\ln \frac{n^{k}}{k !}+1\right)+1= \\
=2^{k} \cdot k \cdot \ln n-\left(2^{k} \cdot \ln (k !)-2^{k}-1\right) .
\end{gathered}
$$

Если $k \geqslant 3$, то $2^{k} \cdot \ln (k !)>2^{k}+1$, поскольку $\ln (k !) \geqslant \ln 6>1,7$ и $2^{k} \cdot \ln (k !)>$ $2^{k} \cdot 1,7>2^{k}+1$. Следовательно, $w(n, k)<2^{k} \cdot k \cdot \ln n$ при $k \geqslant 3$.

Согласно следствию 1 при фиксированном значении $k$ справедливо неравенство $w(n, k)<C_{1} \cdot \ln n$, где $C_{1}>0$ - некоторая константа. Сравним это с нижними оценками из статьи [1]: при фиксированном $k \geqslant 3$ существует такое $n_{0}$, что для всех $n \geqslant n_{0}$ верно $w(n, k)>2^{k-2} \cdot \log _{2} n$ и $w(n, 2) \sim \log _{2} n$. Отсюда следует, что при $k>1$ существует такая константа $C_{2}>0$, что $w(n, k)>C_{2} \cdot \ln n$ при достаточно больших $n$. Значит, доказана асимптотически точная оценка.

Напомним, что для вещественных функций натурального переменного $f(n)$ и $g(n)$ пишут $f(n)=\Theta(g(n))$ при $n \rightarrow \infty$, если существуют такие константы $C_{1}>0$, $C_{2}>0$, что $C_{1} \cdot g(n)<f(n)<C_{2} \cdot g(n)$. Пользуясь этим обозначением, приведем еще одно следствие из утверждения 2.

Следствие 2. Пусть $k>1$ - фиксированное число, тогда $n$ ри $n \rightarrow \infty$

$$
w(n, k)=\Theta(\ln n) .
$$

Очевидно, что способ построения комбинаторно полной матрицы "жадным" алгоритмом покрытия матрицы $T(n, k)$ имеет высокие требования по памяти и трудоемкости. В частности, матрица $T(n, k)$ содержит $2^{n} \cdot\left(\begin{array}{l}n \\ k\end{array}\right)$ единиц, поэтому только для ее хранения потребуется экспоненциальный по $n$ объем оперативной памяти, причем даже для малых значений $k$. Это делает актуальным изучение других подходов к построению комбинаторно полных матриц с возможно меньшим числом строк.

\section{2. Построение комбинаторно полных матриц путем размещения единиц}

в строках. В дальнейшем будем строить комбинаторно полные матрицы порядка $k$ с $n$ столбцами в явном виде для произвольных значений $n, k, n>k>1$. В результате будем получать новые нижние оценки числа строк таких матриц. Эти оценки в общем случае значительно больше оценки, полученной в утверждении 2, за исключением случая, когда $k$ близко к $n$. Достоинство получаемых оценок состоит в их достижимости и конструктивности.

Воспользуемся идеей построения комбинаторно полной матрицы, примененной в [1]. При фиксированных $n, k, n>k>1$, зададим битовую матрицу $Q(n, k)$ с $n$ 
столбцами. Построим эту матрицу итеративным образом. Сначала рассмотрим матрицу $Q^{1}(n, k)$, образованную $m=\left(\begin{array}{l}n \\ k\end{array}\right)$ различными строками длины $n$ веса $k$. В [1] показано, что при $n \geqslant 2 k$ матрица $Q^{1}(n, k)$ будет комбинаторно полной порядка $k$.

Пусть $n<2 k$. В этом случае в произвольной $m \times k$ подматрице матрицы $Q^{1}(n, k)$ строки имеют вес не менее $2 k-n$. Рассмотрим матрицу $Q^{2}(n, k)$, которая получена из матрицы $Q^{1}(n, k)$ добавлением $\left(\begin{array}{c}n \\ 2 k-n-1\end{array}\right)$ новых строк веса $2 k-n-1$. Аналогично предыдущему случаю получаем, что в произвольно выбранных $k$ столбцах матрицы $Q^{2}(n, k)$ стоят все векторы веса не менее $3 k-2 n-1$. Действительно, по координатам, соответствующим не выбранным $n-k$ столбцам, можно разместить $n-k$ единиц, а оставшиеся $2 k-n-1-(n-k)=3 k-2 n-1$ единиц обязательно будут присутствовать в координатах, отвечающих выбранным столбцам. Следовательно, матрица $Q^{2}(n, k)$ - комбинаторно полная порядка $k$ тогда и только тогда, когда $3 k-2 n-1 \leqslant 0$, то есть $n \geqslant \frac{3}{2} k-\frac{1}{2}$.

Если $n<\frac{3}{2} k-\frac{1}{2}$, то рассмотрим матрицу $Q^{3}(n, k)$, полученную из $Q^{2}(n, k)$ добавлением $\left(\begin{array}{c}n \\ 3 k-2 n-2\end{array}\right)$ строк веса $3 k-2 n-2$, и т.д.

При $t \geqslant 2$ матрица $Q^{t}(n, k)$ получается из матрицы $Q^{t-1}(n, k)$ добавлением $\left(\begin{array}{c}n \\ t \cdot k-(t-1) \cdot n-(t-1)\end{array}\right)$ строк веса $t \cdot k-(t-1) \cdot n-(t-1)$. Эта матрица комбинаторно полная тогда и только тогда, когда

$$
t \cdot k-(t-1) \cdot n-(t-1)-(n-k)=(t+1) \cdot k-t \cdot n-(t-1) \leqslant 0 .
$$

Возьмем такое минимальное $t_{0}=t_{0}(n, k)$, при котором неравенство (1) выполнено. То есть $t_{0}=\left\lceil\frac{1+k}{n-k+1}\right\rceil \geqslant 1$ (здесь $\lceil x\rceil$ - наименьшее целое, не меньшее $x)$. Тогда матрица $Q^{t}(n, k)$ - первая матрица в последовательности матриц $Q^{1}(n, k), Q^{2}(n, k), \ldots$, которая является комбинаторно полной порядка $k$. Число строк в этой матрице равно

$$
\sum_{t=1}^{t_{0}}\left(\begin{array}{c}
n \\
t \cdot k-(t-1) \cdot n-(t-1)
\end{array}\right)=\sum_{t=1}^{t_{0}}\left(\begin{array}{c}
n \\
t \cdot(n-k+1) \cdot n-1
\end{array}\right) .
$$

Данное итеративное построение комбинаторно полной матрицы позволяет сформулировать следующий результат.

Утверждение 3. При любых натуралъных $n u k, n>k>1$, верно неравенство

$$
w(n, k) \leqslant \sum_{t=1}^{t_{0}}\left(\begin{array}{c}
n \\
t \cdot(n-k+1) \cdot n-1
\end{array}\right),
$$

где $t_{0}=\left\lceil\frac{1+k}{n-k+1}\right\rceil$.

Указанная оценка весьма грубая, но она для любых значений $n$ и $k$ не превысит $2^{n}$, тогда как в оценке, полученной при помощи "жадного"алгоритма, этот порог может быть превышен. Действительно, при $k=n-c$, где $c$ - константа, и 
растущем значении $n,\left(\begin{array}{l}n \\ c\end{array}\right) \sim \frac{n^{c}}{c !}$, получаем

$$
\begin{aligned}
& {\left[2^{k} \cdot\left(\ln \left(\begin{array}{l}
n \\
k
\end{array}\right)+1\right)\right] \geqslant 2^{k} \cdot\left(\ln \left(\begin{array}{l}
n \\
k
\end{array}\right)+1\right)=} \\
= & \frac{2^{n}}{2^{c}} \cdot\left(\ln \left(\begin{array}{c}
n \\
n-c
\end{array}\right)+1\right)=\frac{2^{n}}{2^{c}} \cdot\left(\ln \left(\begin{array}{l}
n \\
c
\end{array}\right)+1\right)= \\
= & \frac{2^{n}}{2^{c}} \cdot\left(\ln \frac{n^{c}}{c !}+1\right)+1=2^{n} \cdot \ln n \cdot\left(\frac{c}{2^{c}}+o(1)\right)>2^{n} .
\end{aligned}
$$

Однако оценка (3) не всегда лучше, чем для «жадного» алгоритма. Например, для случая $k \leqslant \frac{n}{2}$ сумма (3) равна $\left(\begin{array}{l}n \\ k\end{array}\right)$, что при больших $n$ существенно больше, чем $2^{k} \cdot k \cdot \ln n$ (оценка из следствия 1 ).

Приведем несколько следствий из утверждения 3. Воспользуемся известным результатом о мультисекции суммы биномиальных коэффициентов ([5]).

Следствие 3. При любых натуралъных $n u k, n>k>1$, верно неравенство

$$
w(n, k) \leqslant \frac{1}{n-k+1} \cdot \sum_{j=0}^{n-k}\left(2 \cos \frac{\pi j}{n-k+1}\right)^{n} \cdot \cos \frac{\pi(2 k-n) j}{n-k+1} .
$$

Доказательство. Известно (см. [5]), что при фиксированных $r, s, 0 \leqslant r<s$, верно равенство

$$
\left(\begin{array}{c}
n \\
r
\end{array}\right)+\left(\begin{array}{c}
n \\
r+s
\end{array}\right)+\left(\begin{array}{c}
n \\
r+2 s
\end{array}\right)+\ldots=\frac{1}{s} \cdot \sum_{j=0}^{s-1}\left(2 \cos \frac{\pi j}{s}\right)^{n} \cdot \cos \frac{\pi(n-2 r) j}{s}
$$

В выражение, стоящее в правой части неравенства (3), преобразуем следующим образом:

$$
\begin{aligned}
& \sum_{t=1}^{t_{0}}\left(\begin{array}{c}
n \\
t \cdot(n-k+1)-1
\end{array}\right)=\sum_{t=1}^{t_{0}}\left(\begin{array}{c}
n \\
(t-1) \cdot(n-k+1)+(n-k)
\end{array}\right)= \\
= & \sum_{t=0}^{t_{0}-1}\left(\begin{array}{c}
n \\
t \cdot(n-k+1)+(n-k)
\end{array}\right)=\sum_{t=0}^{\infty}\left(\begin{array}{c}
n \\
t \cdot(n-k+1)+(n-k)
\end{array}\right) .
\end{aligned}
$$

Последний переход верен, так как при $t \geqslant t_{0} \geqslant \frac{1+k}{n-k+1}$ величина $t \cdot(n-k+1)+$ $(n-k) \geqslant n+1$, то есть $\left(\begin{array}{c}n \\ t \cdot(n-k+1)+(n-k)\end{array}\right)=0$.

Положим $r=n-k, s=n-k+1$. Тогда $0 \leqslant r<s$, и, подставляя эти значения в формулу (5), получаем выражение, стоящее в правой части неравенства (4).

Оценим сумму (3) и получим оценку простого вида.

Следствие 4. При любых $n u k, n>k>1$ верно

$$
w(n, k) \leqslant\left(\begin{array}{l}
n \\
k
\end{array}\right), \text { если } k \leqslant \frac{n}{2},
$$




$$
w(n, k) \leqslant\left(\begin{array}{c}
n \\
{[n / 2]}
\end{array}\right) \cdot \frac{n+2}{n-k+1}, \text { если } k>\frac{n}{2} .
$$

Доказательство. Если $k \leqslant \frac{n}{2}$, то $1+k \leqslant n-k+1$ и тогда $t_{0}=\left\lceil\frac{1+k}{n-k+1}\right\rceil=1$. Т.е. в сумме (3) присутствует ровно одно слагаемое, равное $\left(\begin{array}{c}n \\ n-k\end{array}\right)=\left(\begin{array}{l}n \\ k\end{array}\right)$.

Если $k>\frac{n}{2}$, то в сумме $(3)$ всего $t_{0}=\left\lceil\frac{1+k}{n-k+1}\right\rceil<\frac{1+k}{n-k+1}+1=\frac{n+2}{n-k+1}$ слагаемых, поэтому, оценив каждое слагаемое величиной $\left(\begin{array}{c}n \\ {[n / 2]}\end{array}\right)$, получим требуемое неравенство.

3. Построение комбинаторно полных матриц порядков 2 и 3 . Пусть $n=2^{r}, r>0$. Рассмотрим $(0,1)$-матрицу $A$ размера $m \times n$ и будем интерпретировать ее как матрицу, составленную из векторов-строк значений булевых функций $f_{1}, \ldots, f_{m} \in F_{2}(r)$ от $r$ переменных. Столбцы считаем занумерованными $r$-мерными двоичными векторами в лексикографическом порядке. Такую матрицу будем обозначать $A_{F}$, где $F=\left(f_{1}, \ldots, f_{m}\right)$. Очевидна следующая лемма.

Лемма 1. Матрица $A_{F}$ является комбинаторно полной порядка $k$ тогда и только тогда, когда для любых попарно различных векторов $z^{(1)}, \ldots, z^{(k)} \in V_{r}$ и любых значений $c_{1}, \ldots, c_{k} \in\{0,1\}$ найдется такая функиия $f \in F$, что

$$
f\left(z^{(1)}\right)=c_{1}, \ldots, f\left(z^{(k)}\right)=c_{k} .
$$

Для $\alpha=\left(a_{1}, \ldots, a_{r}\right) \in V_{r}, b \in\{0,1\}$ через $<\alpha, x>\oplus b$ обозначим аффинную функцию, задаваемую многочленом Жегалкина $a_{1} x_{1} \oplus a_{2} x_{2} \oplus \ldots \oplus a_{r} x_{r} \oplus b$.

Утверждение 4. Пусть $r \geqslant 1$ u $F=\left\{\langle\alpha, x\rangle \oplus b: \alpha \in V_{r},\|\alpha\| \leqslant 1, b \in\{0,1\}\right\}$. Тогда матрища $A_{F}$ комбинаторно полна порядка 2.

Доказательство. Покажем, что выполнено условие (6) леммы 1. Заметим, что множество функций $F$ можно разбить на два множества: $F=F_{0} \cup F_{1}$, где

$$
F_{0}=\left\{\left\langle\alpha, x>: \alpha \in V_{r},\|\alpha\| \leqslant 1\right\}, F_{1}=\left\{<\alpha, x>\oplus 1: \alpha \in V_{r},\|\alpha\| \leqslant 1\right\} .\right.
$$

Пусть $z^{(1)}, z^{(2)} \in V_{r}, z^{(1)} \neq z^{(2)}, c_{1}, c_{2} \in\{0,1\}$. Поскольку множество $F$ содержит функции-константы, то в случае $c_{1}=c_{2}=0$, либо $c_{1}=c_{2}=1$, условие (6) очевидно выполняется.

Пусть $c_{1} \neq c_{2}$. Поскольку класс $F$ содержит набор функций вместе с их отрицаниями, достаточно рассмотреть случай $c_{1}=0, c_{2}=1$. Положим $z^{(1)}=$ $\left(z_{1}^{(1)}, \ldots, z_{r}^{(1)}\right), z^{(2)}=\left(z_{1}^{(2)}, \ldots, z_{r}^{(2)}\right)$. В силу того, что $z^{(1)} \neq z^{(2)}$, для некоторого $i \in \overline{1, r}$ либо $z_{i}^{(1)}=1, z_{i}^{(2)}=0$, либо $z_{i}^{(1)}=0, z_{i}^{(2)}=1$. В первом случае в качестве функции $f$ выберем функцию $f\left(x_{1}, \ldots, x_{r}\right)=x_{i}$, а во втором - функцию $f\left(x_{1}, \ldots, x_{r}\right)=x_{i} \oplus 1$.

В условиях утверждения очевидно, что $m=2 r+2, r \geqslant 1$.

Данное утверждение позволяет построить комбинаторно полную порядка 2 матрицу размера $m=2 \cdot r+2=2 \cdot \log _{2} n+2 n=2^{r}$. Для значения $n$, не являющегося степенью двойки, применим естественный способ построения: положим $r=\left\lceil\log _{2} n\right\rceil$ и построим комбинаторно полную матрицу порядка 2 с $2^{r}$ столбцами и $m=2 \cdot r+2$ 
строками. Отбросив произвольные $2^{\left\lceil\log _{2} n\right\rceil}-n$ столбцов, получим комбинаторно полную матрицу порядка 2 размера $m \times n$. Поскольку $\lceil x\rceil \leqslant x+1$, то $m \leqslant 2 \cdot \log _{2} n+4$. Известно $([1])$, что $w(n, 2) \sim \log _{2} n$ при $n \rightarrow \infty$. Т.е. в построенной матрице число строк будет с точностью до постоянного множителя совпадет со своим минимально возможным значением.

Описанный подход можно применить к случаю $k=3$.

Утверждение 5. Если $r \geqslant 2 u F=\left\{\left\langle\alpha, x>\oplus b: \alpha \in V_{r},\|\alpha\| \leqslant 2, b \in\{0,1\}\right\}\right.$, mo матрица $A_{F}$ комбинаторно полна порядка 3.

Доказательство. Пусть $z^{(1)}, z^{(2)}, z^{(3)} \in V_{r}$ - различные векторы и $c_{1}, c_{2}, c_{3} \in$ $\{0,1\}$. Согласно лемме 1 достаточно найти такие вектор $\alpha=\left(a_{1}, \ldots, a_{r}\right) \in V_{r}$, $\left\|\alpha^{\prime}\right\| \leqslant 2$, и $b \in\{0,1\}$, что

$$
\begin{aligned}
& <\alpha, z^{(1)}>\oplus b=c_{1}, \\
& <\alpha, z^{(2)}>\oplus b=c_{2}, \\
& <\alpha, z^{(3)}>\oplus b=c_{3} .
\end{aligned}
$$

Поскольку векторы $z^{(1)}, z^{(2)}, z^{(3)}$ попарно различны, найдутся такие координаты $i, j \in\{1,2, \ldots, r\}, i \neq j$, что $z_{i}^{(1)} \neq z_{i}^{(2)}$ и $z_{j}^{(2)} \neq z_{j}^{(3)}$, где $z^{(s)}=\left(z_{1}^{(s)}, \ldots, z_{r}^{(s)}\right), \quad s=$ $1,2,3$. С точностью до перестановки уравнений в (7) и индексов $i$ и $j$ можно считать, что $z_{i}^{(2)}=z_{i}^{(3)}$. Также без ограничения общности можно полагать $i=1, j=2$. Положив $a_{3}=\ldots=a_{r}=0$, из (7) получим систему линейных уравнений от неизвестных $a_{1}, a_{2}, b$, которую запишем в матричном виде:

$$
\left(\begin{array}{ccc}
z_{1}^{(1)} & z_{2}^{(1)} & 1 \\
\bar{z}_{1}^{(1)} & z_{2}^{(2)} & 1 \\
\bar{z}_{1}^{(1)} & \bar{z}_{2}^{(2)} & 1
\end{array}\right) \cdot\left(\begin{array}{c}
a_{1} \\
a_{2} \\
b
\end{array}\right)=\left(\begin{array}{c}
c_{1} \\
c_{2} \\
c_{3}
\end{array}\right)
$$

где для $z=\left(z_{1}, \ldots, z_{r}\right) \in V_{r} \bar{z}=\left(z_{1} \oplus 1, \ldots, z_{r} \oplus 1\right)$.

Матрица системы (8), очевидно, невырожденна при любых $z_{1}^{(1)}, z_{2}^{(1)}, z_{2}^{(2)}$. Поэтому найдутся подходящие значения $a_{1}, a_{2}, b$, удовлетворяющие этой системе. Остается положить $\alpha=\left(a_{1}, a_{2}, 0 \ldots, 0\right)$ для получения равенств $(7)$. По лемме 1 матрица $A_{F}$ является комбинаторно полной порядка 3.

В условиях утверждения 5 построенная матрица $A_{F}$ имеет $m=2 \cdot\left(1+r+\left(\begin{array}{l}r \\ 2\end{array}\right)\right)$ строк и $n=2^{r}$ столбцов. Аналогично случаю $k=2$, перенесем способ построения матрицы на случай произвольного значения $n$. Для этого рассмотрим $r=\left\lceil\log _{2} n\right\rceil$, построим матрицу $A_{F}$, и в ней отбросим $2^{\left\lceil\log _{2} n\right\rceil}-n$ произвольных столбцов. Получим комбинаторно полную матрицу порядка 3 с $n$ столбцами и $m=2 \cdot\left(1+\left\lceil\log _{2} n\right\rceil+\left(\begin{array}{c}\left\lceil\log _{2} n\right\rceil \\ 2\end{array}\right)\right)$ строками. Учитывая, что $\left\lceil\log _{2} n\right\rceil<1+\log _{2} n$, получаем, что $m<2 \cdot\left(2+\log _{2} n+\left(\begin{array}{c}1+\log _{2} n \\ 2\end{array}\right)\right)=\left(\log _{2} n\right)^{2}+3 \cdot \log _{2} n+4$. Таким образом, доказано следствие 5 .

Следствие 5. Для произвольного $n>3$

$$
w(n, 3)<\left(\log _{2} n\right)^{2}+3 \cdot \log _{2} n+4 .
$$




\section{4. Вероятностный метод построения комбинаторно полных матриц.} Для $(0,1)$-матрицы $A$ с $t$ столбцами будем говорить, что строка $\left(x_{1}, \ldots, x_{t}\right) \in V_{t}$ не представлена в матрице $A$, если в матрице $A$ нет строки, равной $\left(x_{1}, \ldots, x_{t}\right)$.

Рассмотрим случайную $(0,1)$-матрицу, в которой каждый элемент принимает значение 0 или 1 случайно, равновероятно и независимо от других элементов. Для $m \times k$-матриц будем говорить об $A_{m, k}$-модели. Если матрица $B=\left(b_{i, j}\right)_{m \times k}$ получена по $A_{m, k}$-модели, то обозначим это $B \in A_{m, k}$.

Пусть $B \in A_{m, k}$. Обозначим через $\eta_{m, k}(B)$ случайную величину, равную числу строк, не представленных в матрице $B$. Очевидно, что $0 \leqslant \eta_{m, k}(B) \leqslant 2^{k}-1$; пусть $p\left(\eta_{m, k}(B)>0\right)$ - вероятность того, что в матрице В представлены не все возможные $2^{k}$ строк. Всего в матрице $B$ имеется $m$ строк, поэтому $p\left(\eta_{m, k}(B)>0\right)=1$ при $m<2^{k}$. Далее считаем, что $m \geqslant 2^{k}$.

Случайную величину $\eta_{m, k}(B)$ можно интерпретировать следующим образом. Векторы из $V_{k}$ - ящики ( $2^{k}$ штук), а строки матрицы $B$ - это дробинки ( $m$ штук). Тогда вероятность попадания $j$-й дробинки (строки с номером $j$ ) в ящик для произвольного фиксированного вектора $\left(x_{1}, \ldots, x_{k}\right) \in V_{k}$ есть

$$
\mathbf{P}\left(b_{1, j}=x_{1}, b_{2, j}=x_{2}, \ldots, b_{k, j}=x_{k}\right)=\prod_{i=1}^{k} \mathbf{P}\left(b_{i, j}=x_{i}\right)=\frac{1}{2^{k}} .
$$

Таким образом, дробинки размещаются по ящикам независимо и равновероятно. Величина $\eta_{m, k}(B)$ в таких терминах есть число пустых ящиков после бросания $m$ дробинок в $2^{k}$ ящиков. Известно (см., например, [6]), что

$$
\mathbf{M} \eta_{m, k}(B)=2^{k} \cdot\left(1-\frac{1}{2^{k}}\right)^{m} .
$$

Рассмотрим случайную величину $\varsigma_{m, k}(B)$, равную 1 , если в матрице $B \in A_{m, k}$ представлены не все возможные $2^{k}$ строк, и $0-$ в противном случае. Тогда $\mathbf{P}\left(\varsigma_{m, k}(B)=0\right)=\mathbf{P}\left(\eta_{m, k}(B)=0\right)$.

Согласно определению матрица $A$ комбинаторно полна порядка $k$ тогда и только тогда, когда любая ее подматрица $\left(A_{i_{1}}^{\downarrow}, \ldots, A_{i_{k}}^{\downarrow}\right)$ не содержит не представленных строк. Рассмотрим матрицу $A$, полученную из $A_{m, n}$-модели, $n \geqslant k$. Обозначим через $q_{m, n, k}$ вероятность того, что матрица $A$ комбинаторно полна порядка $k$. Тогда:

$$
\begin{aligned}
q_{m, n, k} & =\mathbf{P}\left(\varsigma_{m, k}\left(A_{i_{1}}^{\downarrow}, \ldots, A_{i_{k}}^{\downarrow}\right)=0 \forall 1 \leqslant i_{1}<\ldots<i_{k} \leqslant n\right)= \\
& =\mathbf{P}\left(\sum_{1 \leqslant i_{1}<\ldots<i_{k} \leqslant n} \varsigma_{m, k}\left(A_{i_{1}}^{\downarrow}, \ldots, A_{i_{k}}^{\downarrow}\right)=0\right)= \\
& =\mathbf{P}\left(\sum_{1 \leqslant i_{1}<\ldots<i_{k} \leqslant n} \eta_{m, k}\left(A_{i_{1}}^{\downarrow}, \ldots, A_{i_{k}}^{\downarrow}\right)=0\right) .
\end{aligned}
$$

Утверждение 6. Пусть $m, n, k$ - натуральные числа, $k>1, m \geqslant 2^{k}, n \geqslant k$. Тогда

$$
q_{m, n, k} \geqslant 1-\frac{(2 n)^{k}}{k !} \cdot\left(1-\frac{1}{2^{k}}\right)^{m}
$$


Доказательство. Воспользуемся (9) и известным неравенством $\mathbf{P}(\phi>0) \leqslant \mathbf{M} \phi$, верным для произвольной случайной величины $\phi$, принимающей значения $0,1,2, \ldots$. и ее математического ожидания $\mathbf{M} \varphi$ :

$$
\begin{gathered}
1-q_{m, n, k}=1-\mathbf{P}\left(\sum_{1 \leqslant i_{1}<\ldots<i_{k} \leqslant n} \eta_{m, k}\left(A_{i_{1}}^{\downarrow}, \ldots, A_{i_{k}}^{\downarrow}\right)=0\right)= \\
=\mathbf{P}\left(\sum_{1 \leqslant i_{1}<\ldots<i_{k} \leqslant n} \eta_{m, k}\left(A_{i_{1}}^{\downarrow}, \ldots, A_{i_{k}}^{\downarrow}\right)>0\right) \leqslant \mathbf{M} \sum_{1 \leqslant i_{1}<\ldots<i_{k} \leqslant n} \eta_{m, k}\left(A_{i_{1}}^{\downarrow}, \ldots, A_{i_{k}}^{\downarrow}\right) \\
=\sum_{1 \leqslant i_{1}<\ldots<i_{k} \leqslant n} \mathbf{M} \eta_{m, k}\left(A_{i_{1}}^{\downarrow}, \ldots, A_{i_{k}}^{\downarrow}\right)=\sum_{1 \leqslant i_{1}<\ldots<i_{k} \leqslant n} 2^{k} \cdot\left(1-\frac{1}{2^{k}}\right)^{m}= \\
=\left(\begin{array}{c}
n \\
k
\end{array}\right) \cdot 2^{k} \cdot\left(1-\frac{1}{2^{k}}\right)^{m} .
\end{gathered}
$$

Поскольку

$$
\left(\begin{array}{l}
n \\
k
\end{array}\right)=\frac{n \cdot(n-1) \cdot \ldots \cdot(n-k+1)}{k !} \leqslant \frac{n^{k}}{k !},
$$

получим

$$
\left(\begin{array}{l}
n \\
k
\end{array}\right) \cdot 2^{k} \cdot\left(1-\frac{1}{2^{k}}\right)^{m}<\frac{(2 n)^{k}}{k !} \cdot\left(1-\frac{1}{2^{k}}\right)^{m},
$$

откуда следует (10).

Следует сделать важное замечание. При генерации случайной матрицы по $A_{m, n}$-модели со значением $m=k \cdot 2^{k} \cdot \ln n$ при $k \geqslant 3$ получается комбинаторно полная матрица порядка $k$ с вероятностью, стремящейся к 1 с ростом $n$, что примерно соответствует оценке, полученной ранее с помощью «жадного» алгоритма.

При растущем значении $n$ рассмотрим значение числа $m$ строк $(0,1)$-матрицы, как функцию от $n: m(n)=k \cdot 2^{k} \cdot \ln n$. Выделим 3 случая:

$k$ - вещественная функция от $n, k=k(n)=\varepsilon n$, где $0<\varepsilon<1-$ фиксированное вещественное число;

$k$ - функция от $n$ вида $k=k(n)=n-c$, где $0<c-$ фиксированное натуральное число, параметр;

$k$ - фиксированное число, не зависящее от $n$.

Во всех случаях предполагаем, что $n>k>1$. Из утверждения 6 получаем следствие, которое говорит о том, что во всех трех случаях матрица из $A_{m, n}$-модели является комбинаторно полной порядка $k$ с вероятностью, стремящейся к 1.

Следствие 6. Пусть $n$ u $k$-натуральные числа, $n>k>2, m=m(n)=k \cdot 2^{k} \cdot \ln n$ и выполняется любое из условий:

1) $k$ - бункция от $n$ вида $k=k(n)=\varepsilon \cdot n$, где $0<\varepsilon<1$ - фиксированное вещественное число;

2) $k$ - функция от $n$ вида $k=k(n)=n-c$, где $0<c-$ фиксированное натуральное число.

3) $k$ - биксированное число.

Тогда $q_{m, n, k}(A) \rightarrow 1$ nри $n \rightarrow \infty$. 
Доказательство. По формуле (10) имеем:

$$
q_{m, n, k} \geqslant 1-\frac{(2 n)^{k}}{k !} \cdot\left(1-\frac{1}{2^{k}}\right)^{m}=1-\frac{2^{k}}{k !} \cdot n^{k+m \log _{n}\left(1-\frac{1}{2^{k}}\right)} .
$$

Из известной оценки $\ln (1+x) \leqslant x$ при $x>-1$ получаем $\log _{n}\left(1-\frac{1}{2^{k}}\right)<-\frac{1}{\ln n} \cdot \frac{1}{2^{k}}$. Отсюда

$$
q_{m, n, k}>1-\frac{2^{k}}{k !} \cdot n^{k-\frac{m}{\ln n} \cdot \frac{1}{2^{k}}} .
$$

Для условия 1) подставим в (12) $k=\varepsilon \cdot n, m=m(n)=k \cdot 2^{k} \cdot \ln n$ и получим

$$
q_{m, n, k}>1-\frac{2^{\varepsilon \cdot n}}{(\varepsilon \cdot n) !} \cdot n^{0}=1-\frac{2^{\varepsilon \cdot n}}{(\varepsilon \cdot n) !} .
$$

Очевидно, что $\frac{2^{\varepsilon \cdot n}}{(\varepsilon \cdot n) !} \rightarrow 0$ при $n \rightarrow \infty$, поэтому $q_{m, n, k} \rightarrow 1$.

Для условия 2) подставим в (12) $k=n-c, m=m(n)=k \cdot 2^{k} \cdot \ln n$ и получим

$$
\begin{gathered}
q_{m, n, k}>1-\frac{2^{n-c}}{(n-c) !}=1-\frac{1}{2^{c}} \cdot \frac{2^{n}}{(n-c) !} \\
=1-\frac{1}{2^{c}} \cdot \frac{2^{n} \cdot(n-c+1) \cdot(n-c+2) \cdot \ldots \cdot n}{n !}>1-\frac{1}{2^{c}} \cdot \frac{2^{n} \cdot n^{c}}{n !} .
\end{gathered}
$$

Очевидно, что $\frac{2^{n} \cdot n^{c}}{n !} \rightarrow 0$ при $n \rightarrow \infty$, поэтому $q_{m, n, k} \rightarrow 1$.

Для условия 3) в формуле (11) оценим сверху величину $\log _{n}\left(1-\frac{1}{2^{k}}\right)$. Имеем: $\log _{n}\left(1-\frac{1}{2^{k}}\right)=\frac{\ln \left(1-\frac{1}{2^{k}}\right)}{\ln n}$. После разложения в ряд Тейлора имеем:

$$
\ln \left(1-\frac{1}{2^{k}}\right)=-\frac{1}{2^{k}}-\frac{1}{2} \cdot \frac{1}{2^{2 k}}-\frac{1}{3} \cdot \frac{1}{2^{3 k}}-\ldots<-\left(\frac{1}{2^{k}}+\frac{1}{2^{2 k+1}}\right) .
$$

Тогда

$$
\log _{n}\left(1-\frac{1}{2^{k}}\right)<-\frac{1}{\ln n} \cdot\left(\frac{1}{2^{k}}+\frac{1}{2^{2 k+1}}\right) .
$$

В итоге из (11) следует, что

$$
q_{m, n, k}(A)>1-\frac{2^{k}}{k !} \cdot n^{k-\frac{m}{\ln n}\left(\frac{1}{2^{k}}+\frac{1}{2^{2 k+1}}\right)} .
$$

Подставляем $m=m(n)=k \cdot 2^{k} \cdot \ln n$ и получаем

$$
q_{m, n, k}(A)>1-\frac{2^{k}}{k !} \cdot n^{k-k \cdot 2^{k}\left(\frac{1}{2^{k}}+\frac{1}{2^{2 k+1}}\right)}=1-\frac{2^{k}}{k !} \cdot n^{-\frac{k}{2^{k+1}}}
$$

Так как при $n \rightarrow \infty$ и фиксированном значении $k$ функция $n^{-\frac{k}{2^{k+1}}} \rightarrow 0$, то $q_{m, n, k} \rightarrow 1$. 


\section{Список литературы}

1. Ролдугин П.В., Тарасов А.В., "Функции без коротких имплицент. Часть I: нижние оценки весов", Дискретная математика, 27:2 (2015), 94-105.

2. Глушков В.М., Синтез иифровых автоматов, М.: ГИФМЛ, 1962.

3. Сачков В.Н., Тараканов В.Е., Комбинаторика неотрицательных матрии, М.: ТВП, 2000.

4. Райгородский А. М., Системы общих представителей и их приложения в геометрии, М.: МЦНМО, 2006.

5. Риордан Дж., Комбинаторные тождества, М.: Наука, 1982.

6. Колчин В.Ф., Севастьянов Б.А., Чистяков В.П., Случайные размещения, М.: Наука, 1976.

Статья поступила 27.01.2015. 